\title{
Shock-induced mixing of a light-gas cylinder
}

\author{
By J. W. JACOBS $\dagger$ \\ California Institute of Technology, Pasadena, CA 91125, USA
}

(Received 14 September 1990 and in revised form 18 June 1991)

\begin{abstract}
Experiments have been carried out to quantify the mixing induced by the interaction of a weak shock wave with a cylindrical volume of a gas (helium) that is lighter than its surroundings (air). In these experiments a round laminar jet was used to produce the light-gas cylinder, and planar laser-induced fluorescence (PLIF), utilizing a fluorescent tracer (biacetyl) mixed with the helium, was used to visualize the flow. These techniques provide a higher quality of flow visualization than that obtained in previous investigations. In addition, the PLIF technique could be used for the measurement of species concentration. The distortion of the helium cylinder produced by the passing shock wave was found to be similar to that displayed by images from previous experimental and computational investigations. The downstream displacement of several points on the boundary of the light-gas cylinder are measured and agree reasonably well with the results of earlier experimental and theoretical studies as well. Because the mixing process causes the helium originally contained within the cylinder to be dispersed into the surrounding air, the PLIF image area inside the contour at one half the maximum concentration of the fluorescent tracer decreases as the two gases mixed. The change in this area is used as a measure of the mixing rate, and it is found that the time rate of change of this area divided by the area of the initial jet is approximately $-0.7 \times 10^{3} \mathrm{~s}^{-1}$.
\end{abstract}

\section{Introduction}

When two motionless gases are brought into contact, they mix by diffusion, at a rate proportional to the product of the contact area and the concentration gradient at that interface. If the gases are set into motion, this mixing rate will increase, simply because the motion produces an increase in the contact area. For example, small-scale turbulent motion can distort or even break apart the interface, greatly increasing the contact area, and thus, the mixing rate. However, fluid motion can further enhance mixing by altering interfacial concentration gradients. Marble (1985) has shown that the motion induced by a potential vortex enhances diffusion in the mixing of fuel and oxidizer in a laminar flame, by stretching the interface that separates the two gases. Interfacial stretching, in effect, creates new surface by pulling away already mixed fluid, while simultaneously drawing fresh unmixed fluids toward the interface. The net result of this motion is to steepen concentration gradients at the interface, and thus, accelerate diffusion and mixing. In another example, Broadwell \& Breidenthal (1982) maintain that at least part of the mixing that occurs in a turbulent mixing layer, takes place in strained laminar diffusion layers, located between the large vortical structures that are the dominant feature in this flow. In this case, motion induced by vorticity contained within the large

$\dagger$ Present address: Department of Aerospace and Mechanical Engineering, University of Arizona, Tucson, AZ 85721, USA. 
structures acts to stretch the interface that separates the fluids entrained from either side of the shear layer, and therefore, enhances the mixing rate in these regions.

Vorticity plays an important role in the mixing of many free shear flows; however, vorticity need not be the result of the merging of two streams of different velocity. It is well known that vorticity can be produced by the misalignment of pressure and density gradients. For example, the vorticity in buoyant flows is produced by the interaction of a hydrostatic pressure gradient with a density difference, though the amount of vorticity produced in these situations is often not large enough to produce vigorous mixing. On the other hand, the interaction of a density gradient with the pressure gradient from a shock wave can generate high concentrations of vorticity, and therefore, may produce large mixing rates.

The study of the interaction of shock waves and density gradients began with work concerned with the stability of an accelerated system of gases with unequal densities. The stability of two gases separated by a flat interface, and accelerated by a shock wave travelling normal to the interface was first studied analytically by Richtmyer (1960) and experimentally by Meshkov (1969). In this work it was discovered that small undulations on the nearly flat interface grow with time, when acted on by the passing shock wave. This behaviour was found to be related to Rayleigh-Taylor instability, with the shock wave supplying an impulsive acceleration to the system. Work on this subject has continued both in the United States (Brouillett \& Sturtevant 1988; Saffman \& Meiron 1989) as well as in the Soviet Union (Zaitsev et al. 1985). A variation on this configuration has been investigated by Bonazza et al. (1985) and Yang, Zabusky \& Chen (1990), who have studied the interaction of a plane shock wave with a system of two gases separated by a flat interface that is inclined to the incident shock wave.

Experimental investigations of the interaction of shock waves with curved interfaces began with Markstein (1957a,b) and Rudinger (1958), who studied the interaction of a shock wave with a flame front having a roughly spherical shape. Rudinger \& Somers (1960) later studied the more fundamental problem of the interaction of a plane shock wave with light- or heavy-gas spherical or cylindrical inhomogeneities, produced using either spark discharges or a small jets of $\mathrm{H}_{2}, \mathrm{He}$ or $\mathrm{SF}_{6}$. More recently Haas \& Sturtevant (1987) have reported experiments in which the interaction of a shock wave with light- and heavy-gas cylindrical and spherical inhomogeneities is beautifully visualized. In this work the spherical shapes were produced using soap bubbles filled with either a light or a heavy gas, while the cylindrical inhomogeneities were encapsulated in thin nitrocellulose membranes. Since then a number of researchers have worked to computationally reproduce the results of Haas \& Sturtevant (Picone et al. 1985; Picone \& Boris 1988; Cowperthwaite 1989).

The present investigation focuses on the flow produced by the interaction of a weak shock wave with a cylindrical region of gas that is lighter than its surroundings. Previous experimental investigations have utilized shadowgraph or Schlieren visualization, and have been limited by the integrating nature of these methods to effectively visualize three-dimensional flows. Because shadowgraph and Schlieren images are two-dimensional projections of a three-dimensional density field, all depth information along the optical axis is lost. Furthermore, small non-uniformities along the optical axis tend to block light transmission; and when the light path is long, these obstructions can accumulate, and mask large portions of the image. Haas \& Sturtevant (1987) used shadowgraph visualization oriented along the axis of a helium-filled cylindrical membrane; however, the flow that they encountered had a 
significant amount of small-scale three-dimensionality. The blockage resulting from small-scale interfacial variations caused the interface in their shadowgraph images to appear to be much thicker than it actually was, and as a result, small interfacial detail was lost.

The shortcomings of shadowgraph visualization are overcome in the present work by using planar laser-induced fluorescence (PLIF). In the present experiments helium is seeded with a small amount of the biacetyl (Epstein 1977), and then made to fluoresce with a sheet of laser light. Thus, cross-sectional views of the threedimensional density field are obtained. Biacetyl is a gaseous fluorescent dye with an absorption peak at $425 \mathrm{~nm}$ and a fluorescence peak at $460 \mathrm{~nm}$. The fluorescence of biacetyl has been found to be fairly independent of temperature and insensitive to quenching (Epstein 1974), making the intensity of fluorescence linearly dependent on only the incident radiation and dye concentration. Thus, the PLIF system could be used to measure species concentration. The visualization of Haas \& Sturtevant (1987) was further complicated by interference from the nitrocellulose membrane they used to encapsulate their cylinders. In the present experiments, this effect is removed by employing a new technique in which a laminar helium jet is used to produce the light-gas cylinder, thus eliminating the need for a membrane to contain the lighter gas. Not only does this technique improve the quality of visualization, but it also removes the possibility of the membrane affecting the flow on a larger scale.

\section{Experimental apparatus and procedure}

The present experiments were performed in the Galcit 17 in. shock tube (Liepmann et al. 1962). The $43.18 \mathrm{~cm}$ diameter tube was fitted, for these experiments, with a $60.96 \mathrm{~cm}$ long, $26.67 \mathrm{~cm}$ square test section, with the transition from round shock tube to square test section accomplished using a $152.4 \mathrm{~cm}$ long 'cookie cutter' (figure 1). A pair of $15.24 \mathrm{~cm}$ diameter round windows provided visual access to the test section, and pressure taps fitted with piezoelectric pressure transducers provided timing signals needed to measure shock strength as well as to synchronize the experiments. Shock Mach numbers $\left(M_{\mathrm{s}}\right)$ of approximately 1.093 and 1.15 were produced using aluminium diaphragms, having $0.152 \mathrm{~mm}$ and $0.254 \mathrm{~mm}$ thicknesses, respectively.

A round laminar helium jet was introduced into the test section through a section of $\frac{3}{8}$ in. ( $0.794 \mathrm{~cm}$ inside diameter) copper tubing that entered the shock tube through the sidewall of the test section, and terminated in the central portion of the test section, allowing the jet to emit vertically upward, within the view of the lower window. A second piece of tubing, having a $1.9 \mathrm{~cm}$ opening, was situated $8.9 \mathrm{~cm}$ directly above the jet exit, to collect the light gas and remove it from the shock tube, thus ensuring that excess helium would not accumulate in the test section. An ejector-type vacuum pump was used to remove the excess gas, with a rotameter to monitor the flow rate. Helium was supplied from high-pressure bottles, and flowed through a pair of pressure regulators and a needle valve before it split into two streams. One stream was bubbled through liquid biacetyl, seeding the helium with nearly saturated vapour, while both streams passed through rotameters to measure flow rates, and needle valves to control the proportion of gas flow in each stream, then merged again before entering the test section. A ratio of 10 parts pure helium to 1 part biacetyl-laden helium was used in the experiments, which guaranteed that the addition of biacetyl to the helium stream would not increase the mixture density by more than $10 \%$. 


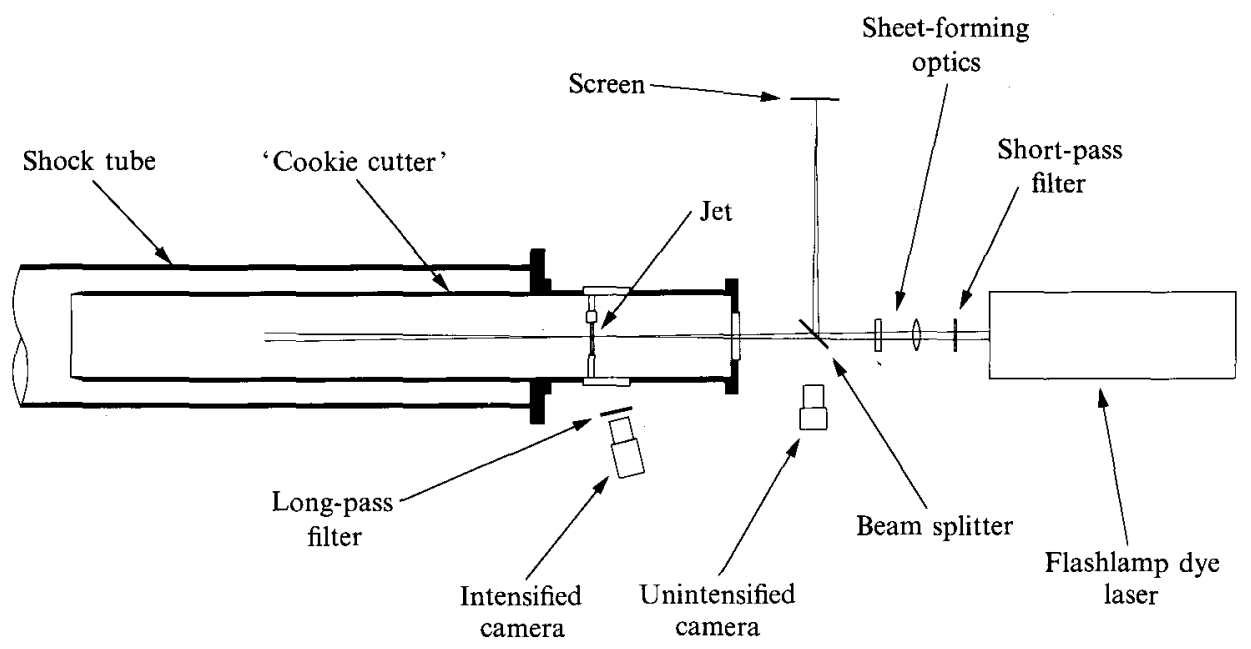

Figure 1. Side view of the experimental apparatus showing the laser-induced fluorescence system.

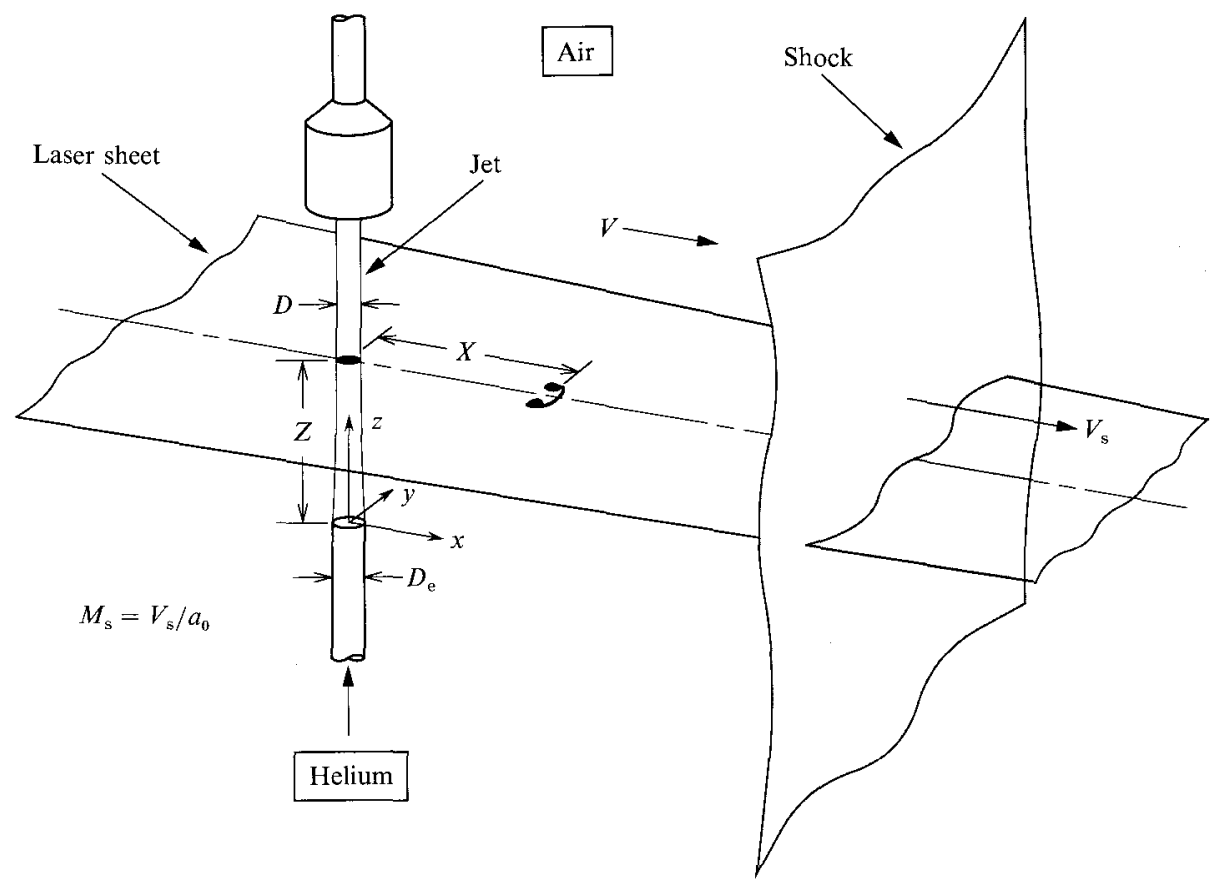

Figure 2. The jet/PLIF configuration.

Planar laser-induced fluorescence was the primary flow visualization technique used in these experiments. A Candella SLL-1050M flashlamp pumped dye laser, filled with Exciton LD423 laser dye, provided $100 \mathrm{~mJ} 0.7 \mu$ s pulses of $430 \mathrm{~nm}$ laser light. The resulting $18 \mathrm{~mm}$ beam was first passed through a $450 \mathrm{~nm}$ short-pass filter, then focused using a set of circular and cylindrical lenses to provide a sheet of laser light that bisected the biacetyl-seeded helium jet, illuminating a $1 \mathrm{~mm}$ thick cross-section (figure 2). The resulting fluorescent image was captured using a Xybion model ISG03 intensified CID camera, positioned so that it viewed upward through the lower 
test section window. The camera output was sent to a Poynting Products PC-170 frame grabber housed in an IBM PC/AT laboratory computer. The camera was fitted with a Schott GG 455 optical glass long-pass filter to eliminate stray laser light within its view. The image intensifier was operated in a gated mode, giving the camera the equivalent of a $3 \mu$ s exposure time. Because the flow behind the passing shock wave pushes the jet down the shock tube, the intensified camera had to be precisely positioned at the location where the jet was expected to be, at the instant the laser was fired. This was accomplished by mounting the intensified camera on a Velmex Unislide linear translator, which allowed the camera to be positioned to within $0.01 \mathrm{~cm}$ of the desired downstream position.

Since the laser pulses had poor shot-to-shot repeatability, a system was implemented to calibrate laser sheet intensity. Immediately after passing through the focusing optics, and before entering the test section, the laser beam passed through a $70 / 30$ beam splitter placed at roughly a $45^{\circ}$ angle. This diverted a portion of the beam $(30 \%)$, projecting a duplicate image of the sheet onto a screen placed outside of the test section. The resulting image was then viewed with an ordinary (unintensified) General Electric TN2505 CID camera, and captured by a second frame grabber housed in the PC/AT. This allowed for the acquisition of an image of the laser sheet each time the laser was fired, providing pairs of images which were used to measure biacetyl concentrations. The unintensified camera viewed the projected image of the laser sheet through the beam splitter. Thus, a reflected view of the interior of the test section (including the jet) was superimposed onto the image of the laser sheet; and, when a grid was placed in the test section at the jet location, a one to one correspondence of laser sheet intensity to test section location could easily be obtained.

The PLIF system utilized biacetyl vapour as a fluorescent tracer mixed with the helium stream. Because the fluorescence of biacetyl is linearly dependent only on the incident radiation and dye concentration, the PLIF system could be used to measure species concentration. However, because of the low signal-to-noise ratio of the camera (approximately 10), and the fact that the amount of biacetyl introduced into the helium stream could not be carefully controlled, concentration measurements were not precise. Helium was seeded with biacetyl, in these experiments, with the intent of using the fluorescent dye to tag the helium. However, helium and biacetyl diffuse through air at much different rates. The diffusivity of helium in air is $0.69 \mathrm{~cm}^{2} / \mathrm{s}$, while, the diffusivity of a biacetyl in air is approximately $0.089 \mathrm{~cm}^{2} / \mathrm{s}$. Thus, helium diffuses through air at roughly 8 times the rate of biacetyl. Because diffusion lengths are proportional to the square root of the diffusivity (Marble 1985), the diffusion thickness of helium will be roughly 3 times the diffusion thickness of the tracer gas.

Shadowgraphs were used in addition to PLIF to visualize the flow. The shadowgraph system consisted of a Nanolamp spark source, two concave mirrors and two flat mirrors (figure 3 ). The projected shadowgraph images were captured using the unintensified CID camera with the frame grabber used in the PLIF experiments. The system was oriented horizontally, so that the full length of the jet could be viewed. To accomplish this, the test section had to be rotated $90^{\circ}$ from its position in the PLIF experiments, thus making the acquisition of simultaneous PLIF and shadowgraph images impossible.

The shock tube was not configured to be fired on demand; thus, it was necessary to trigger the laser and the data acquisition systems using pressure signals produced by the incident shock wave. PCB model $112 \mathrm{~A} 2$ piezoelectric transducers mounted 


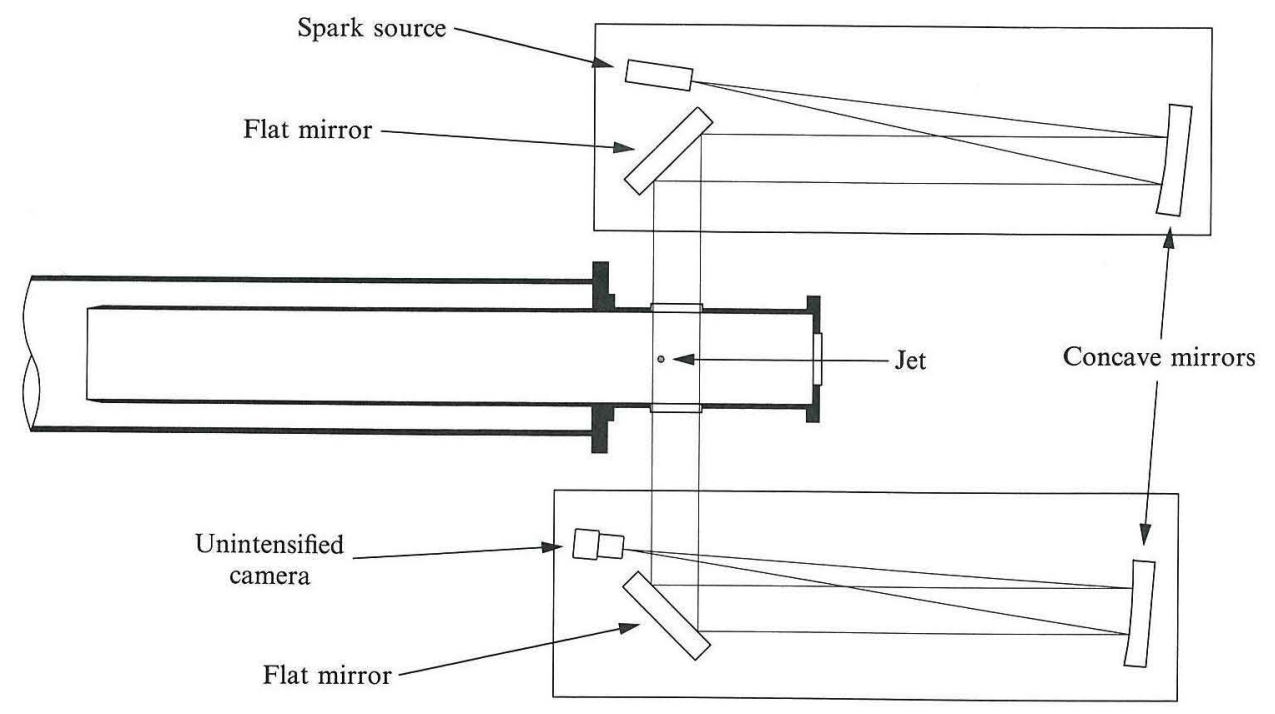

Figure 3. Top view of the experimental apparatus showing the spark shadowgraph system.

flush in the test section walls provided these signals. The transducer output was fed to two Stanford Research DG535 digital delay generators, which produced the delayed signals necessary for the coordination of the laser firing, frame grabber triggering and camera gating. In addition, the pressure signals were recorded using an RC Electronics Computerscope A/D interface installed in a second IBM PC/AT computer.

As described above, calibration of the PLIF system was achieved by placing a grid in the plane of the jet axis, within view of both the intensified and unintensified cameras. After an image of the grid was grabbed and stored, the grid was removed and the shock tube was sealed. The helium flow was then turned on, and images were acquired of the jet cross-section, and the corresponding laser sheet that produced the PLIF image of the jet. A time delay was then programmed into the delay generators, and the intensified camera was placed at the position where the jet was predicted to be, at that time delay. Next, the frame grabber and A/D converter programs were armed, and a diaphragm was positioned into the shock tube. Finally, the shock tube was pressurized, breaking the diaphragm and sending a shock wave travelling down the shock tube.

\section{Results and discussion}

A jet of helium was used in these experiments to produce a light-gas cylinder; thus it was necessary to produce a laminar jet in the test section that was as free as possible from non-uniformities. The shadowgraph system provided a view of the entire length of the jet; therefore it proved to be a useful tool to observe the jet as the helium flow rate and other conditions were varied. It was quickly determined that there was a narrow range of flow velocities that produced laminar jets of sufficient uniformity for this study, and flow rate of $65.1 \mathrm{~cm}^{3} / \mathrm{s}$ was chosen from this range. This flow rate corresponds to a jet Reynolds number of 86 , based on the jet exit diameter and the viscosity of helium. It was found that when the helium flow rate was increased above this value, waves appeared on the jet. On the other hand, when the helium flow rate was decreased significantly below $65.1 \mathrm{~cm}^{3} / \mathrm{s}$, the jet would 
(a)

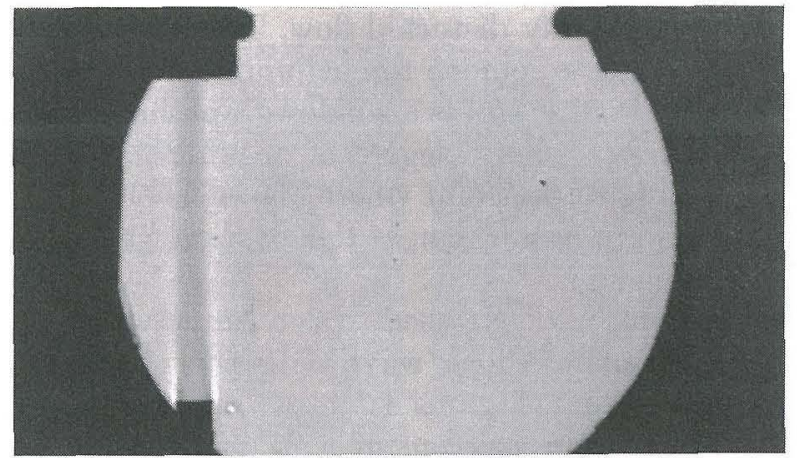

(b)

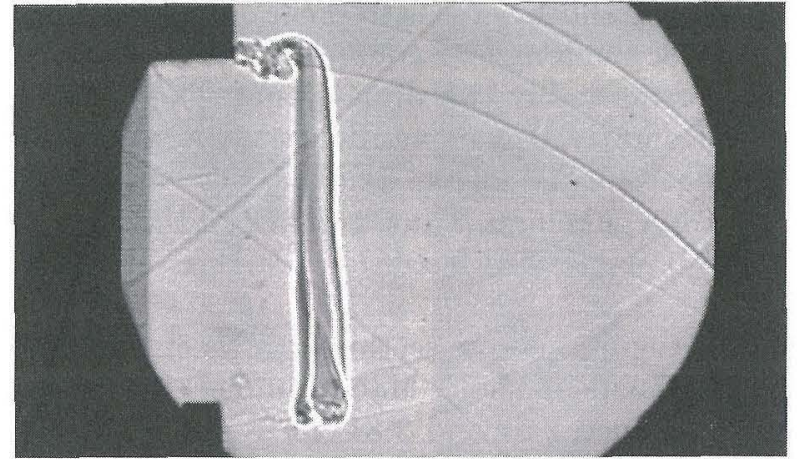

(c)

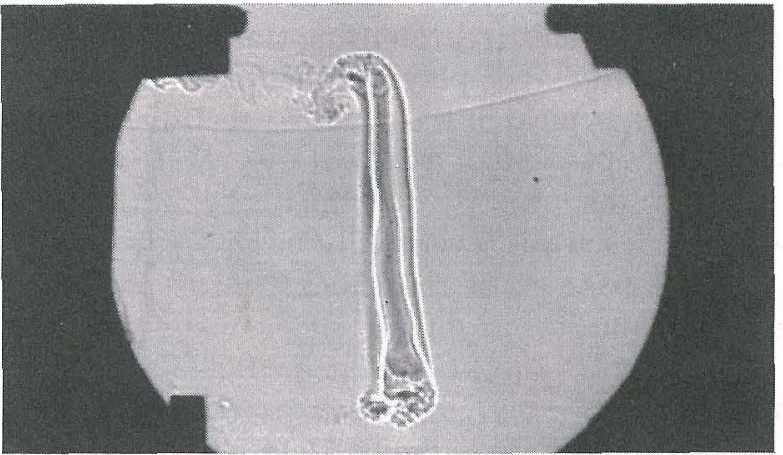

(d)

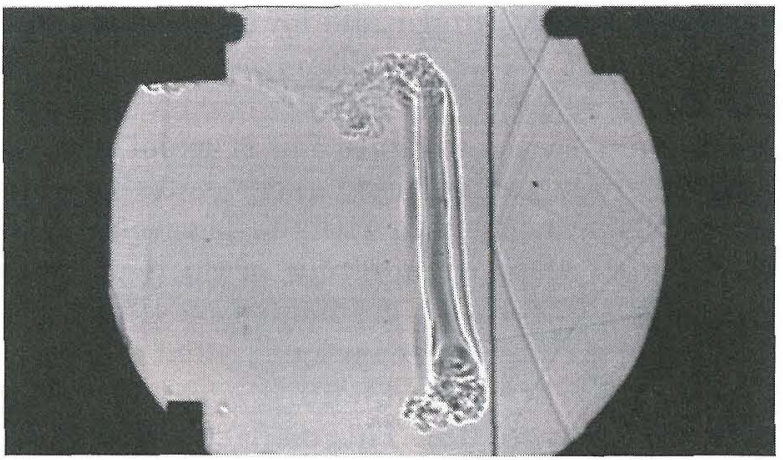

FIgURE 4. A sequence of spark shadowgraph images giving a side view of the evolving helium inhomogeneity. (a) The initial jet, $(b) t=0.435 \mathrm{~ms}$ after the passage of the shock wave, $(c) t=$ $0.921 \mathrm{~ms},(d) t=1.171 \mathrm{~ms}$. The $M_{\mathrm{s}}=1.093$ shock wave has passed from left to right and is shown in the last photograph after reflecting off the back wall of the test section. The images were taken from 3 separate runs. 
break up into an unsteady highly distorted flow. This exploration also showed the magnitude of suction applied to remove the helium stream to have no visible effect on the stability of the jet. Figure $4(a)$ is a shadowgraph image of a typical jet. Note that the buoyant jet shows a slight degree of necking near the jet exit. The jet diameter $(D)$, corresponding to the point where biacetyl concentration is one half its centreline value, was found to be $0.66 \mathrm{~cm}$, at the location where the PLIF views were obtained.

The shadowgraph system was next used to determine whether the flow resulting from the interaction of a plane shock wave with the nearly two-dimensional jet remains nearly two-dimensional. Figures $4(b), 4(c)$ and $4(d)$ are photographs taken $0.435,0.921$ and $1.171 \mathrm{~ms}$ after passage of a $M_{\mathrm{s}}=1.093$ shock wave. The most notable effect of the passing shock wave is to shear off a segment of the jet, and push it downstream. Except for small regions confined to the ends of this jet segment, the flow appears to remain remarkably two-dimensional. The pictures do show that the cylinder becomes tilted slightly as it moves downstream, carried by the flow behind the shock wave. This effect is caused by an axial density variation in the jet, resulting from the outward diffusion of helium. Thus, portions of the jet closer to the exit (and of slightly lower density) are pushed faster (and farther downstream) than sections farther from the exit.

Next, PLIF was used to obtain cross-sectional views of the helium cylinder. These runs were made at two incident shock Mach numbers $\left(M_{\mathrm{s}}=1.093\right.$ and 1.15$)$ and with the laser sheet positioned 2.54 and $3.81 \mathrm{~cm}$ from the jet exit $\left(Z / D_{\mathrm{e}}=3.2\right.$ and 4.8). Figure 5 (plate 1 ) shows a series of flourescent images taken from these runs, displayed in false colour with white representing the lowest fluorescent intensity, and yellow the highest. Figures $5(a-h)$ display a sequence of images taken from 7 separate runs, along with a view of a typical initial jet cross-section (figure $5 \alpha$ ). All were taken at different times after the passage of a $M_{\mathrm{s}}=1.093$ shock wave, and at $Z / D_{\mathrm{e}}=3.2$. The sequence illustrates the process by which the initially circular cylinder is distorted by a passing shock wave, eventually becoming a vortex pair. Figure $5(b)$, taken $0.123 \mathrm{~ms}$ after the passage of the shock, shows an initial flattening of the cylinder caused by the compression of the shock wave, and the beginning of the formation of two lobes. Figure $5(c)$, taken at $t=0.273 \mathrm{~ms}$, shows further development of these lobes, and the beginning of their separation. In figure $5(d)$, the thin strand that connects the two halves in figure $\mathbf{5}(c)$ is stretched and nearly disappears. In figure 5(e), the nearly separated upper and lower halves begin to split into front and back lobes. Figure $5(f-h)$ shows the disappearance of the rearward portions resulting from that split.

In describing the motion observed in figure 5, it is useful to consider the dynamics of vorticity. Vorticity is produced in these experiments by the interaction of the pressure gradient from a plane shock wave with the density gradient at the boundary of a circular cylinder (figure $6 a$ ). The magnitude of this vorticity is proportional to the product of the density and pressure gradients as well as to the degree of their misalignment. Thus, the vorticity produced is localized at the cylinder boundary and is distributed with maxima at the top and bottom edges of the cylinder, and is zero at the front and back edges (figure $6 b$ ). This distribution is similar to that of two semicircular vortex sheets of opposite sign, with a vortex strength distribution that is maximum at the centre of the sheets, and decays towards the ends. Since very little vorticity is produced after the shock passes the jet, the evolution of figure 5 can be described by the dynamics of these two vortex sheets. In figure $5(b-h)$ the vortex sheets are observed to roll up onto themselves, slicing through the helium, and 


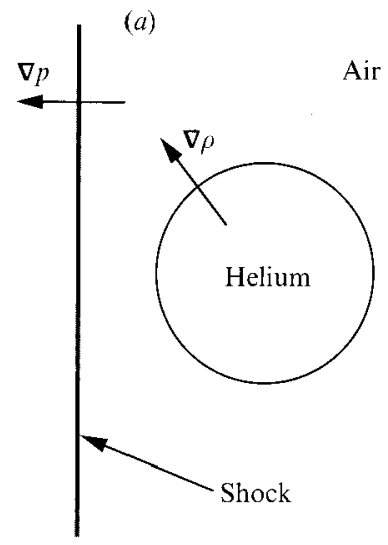

(b)

Air

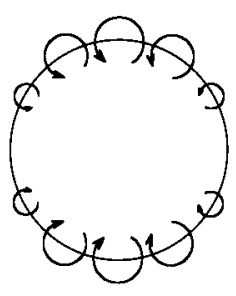

FIGURE 6. The vorticity distribution produced by the interaction of a shock wave with a helium cylinder. (a) The pressure and density gradients, $(b)$ the resulting vorticity distribution.

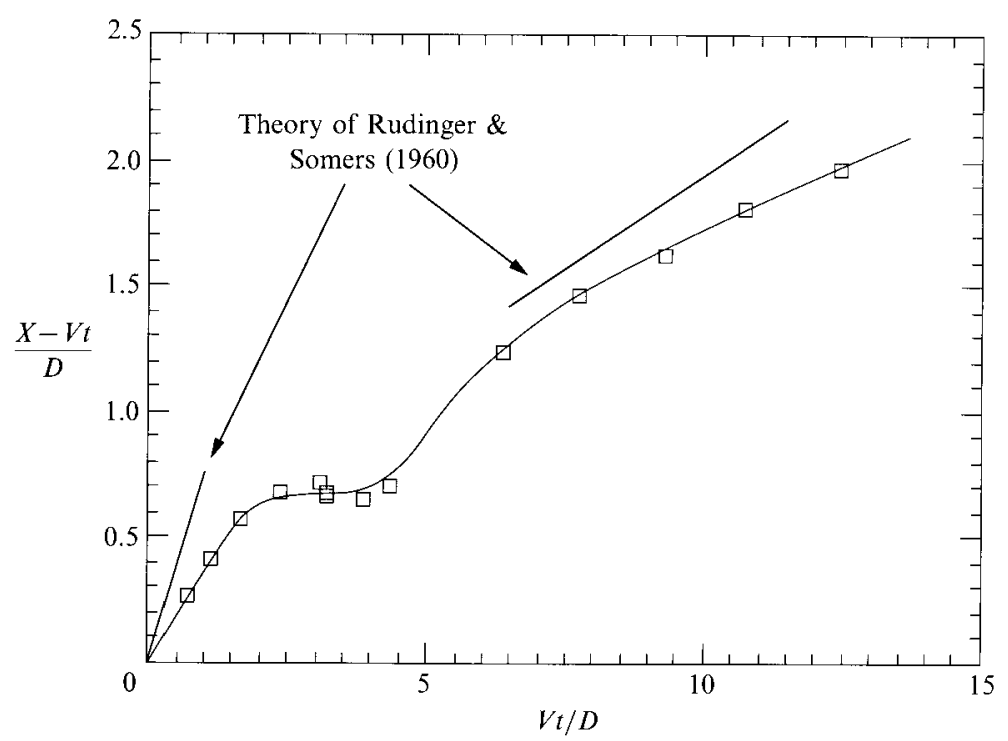

Figure 7. Displacement of the centroid of the helium inhomogeneity relative to the free stream, for the set of runs with $M_{\mathrm{s}}=1.093$ and $Z / D_{\mathrm{e}}=4.8$.

chopping the cylinder into first two and then four pieces as the vorticity spirals inward to form a vortex pair.

\subsection{Displacement}

As the shock wave travels down the shock tube, it accelerates the air that it travels through from rest to a uniform velocity $V$ given by

$$
V=\frac{2 a_{0}}{\gamma+1}\left(M_{\mathrm{s}}-\frac{1}{M_{\mathrm{s}}}\right),
$$

where $\gamma$ is the ratio of specific heats, and $a_{0}$ is the speed of sound preceding the shock. Because helium is lighter than air, the cylinder is accelerated to a velocity greater than $V$. Thus the light gas moves ahead of the surrounding air, as it is carried down the shock tube behind the incident shock wave. Initially, the helium is translated as 


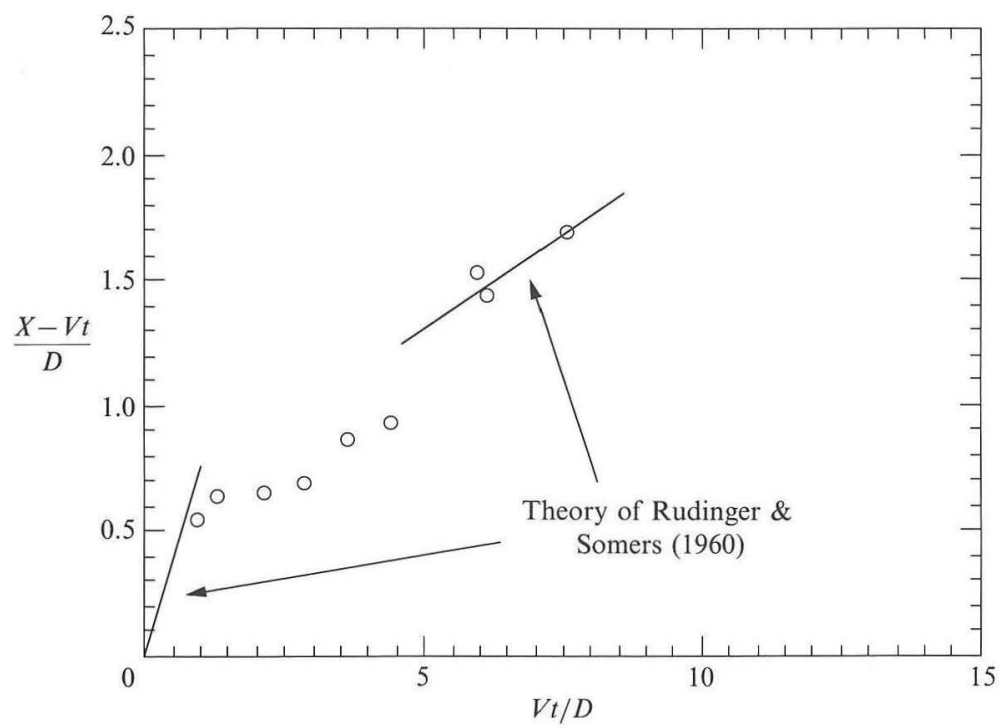

Figure 8. Displacement of the centroid of the helium inhomogeneity relative to the free stream, for the set of runs with $M_{\mathrm{s}}=1.093$ and $Z / D_{\mathrm{e}}=3.2$.

a solid cylinder. However, the light gas is unable to resist the forces caused by the motion of the surrounding air, and is forced to deform, eventually becoming a vortex pair. Figure 7 is a plot of the downstream displacement of the light-gas volume $(X)$, relative to the displacement of an air particle behind the shock wave $(V t)$, for the set of runs with $M_{\mathrm{s}}=1.093$ and $Z / D_{\mathrm{e}}=4.8$. The displacement is that of the centroid of the portion of the PLIF image that is brighter than one half the brightest point in that image, and is scaled with jet diameter $D$. Time in this plot is scaled with $D / V$, the time it takes a free-stream particle to travel one jet diameter. The slope of a line passing through the data is equal to the velocity of the helium cylinder relative to the free-stream velocity. Thus, the light-gas volume initially travels at its greatest velocity, then slows to nearly a standstill, accelerating again to a final velocity that is less than its initial value.

Rudinger \& Somers (1960) presented a simple analysis to describe the displacement of a shock-accelerated cylindrical inhomogeneity. In their analysis, the flow is divided into two regimes. In the first, they assume that the inhomogeneity behaves, initially, like a solid cylinder, the density of which is equal to the density of helium. In the second regime, they assume that the inhomogeneity evolves into a vortex pair with the hydrodynamic impulse and circulation of an infinite lamina accelerated from rest. From this analysis, the initial velocity of the cylinder relative to the surrounding gas is given by

$$
U_{\mathrm{i}}=\frac{1-\sigma}{1+\sigma} V
$$

and the final velocity of the vortex pair is given by

$$
U_{\mathrm{f}}=\frac{2}{\pi^{2}} U_{\mathrm{i}}
$$

Here $\sigma$ is the ratio of cylinder to surrounding gas density, which for a helium/air system equals 0.138 . Substituting this value into (2) and (3) one finds $U_{i}=0.76 \mathrm{~V}$ and 
(a)

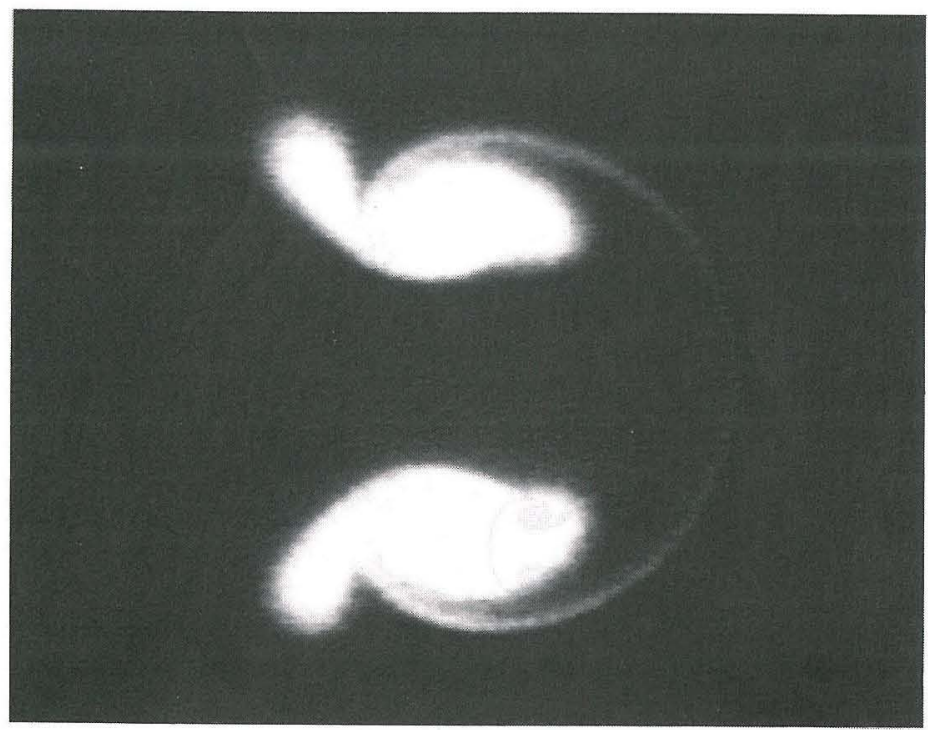

(b)

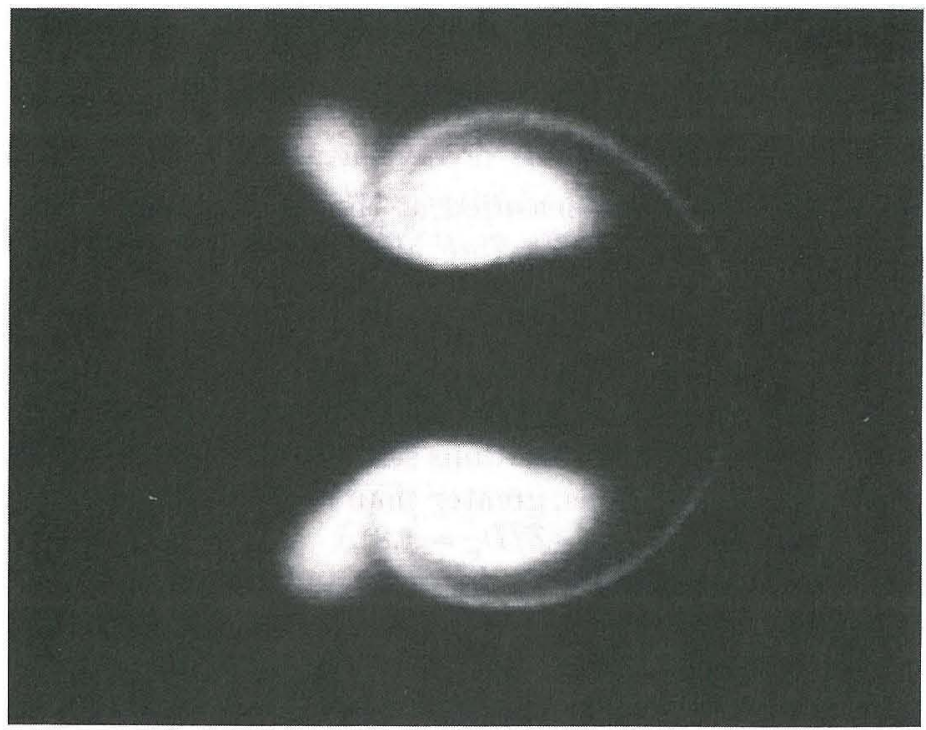

Figure 9. Two cross-sectional views showing a similar degree of evolution, but taken at different cross-sectional locations and at different times. $(a) Z / D_{\mathrm{e}}=3.2$ and $t=0.473 \mathrm{~ms},(b) Z / D_{\mathrm{e}}=4.8$ and $t=0.568 \mathrm{~ms} . M_{\mathrm{s}}=1.093$.

$U_{\mathrm{f}}=0.15 \mathrm{~V}$. Note that this analysis assumes inviscid incompressible flow; thus, $U_{\mathrm{i}}$ and $U_{\mathrm{f}}$ are functions only of the density ratio, $\sigma$, and the velocity jump across the shock, $V$.

Drawn on figure 7 are two lines with slopes corresponding to the initial and final velocities given by (2) and (3). Although the theory significantly overpredicts the experimental velocities both in the initial and final stages, agreement with the experimental data is reasonable. Figure 8 is a plot similar to figure 7 , except with data taken at a station closer to the jet exit $\left(Z / D_{\mathrm{e}}=3.2\right)$. Despite the sparsity of the data in figure 8 , the theory appears to be in better agreement with measurements taken closer to the jet exit. Recall that the shadowgraph images described earlier revealed that the inhomogeneity becomes tilted as it is swept down the shock tube. 


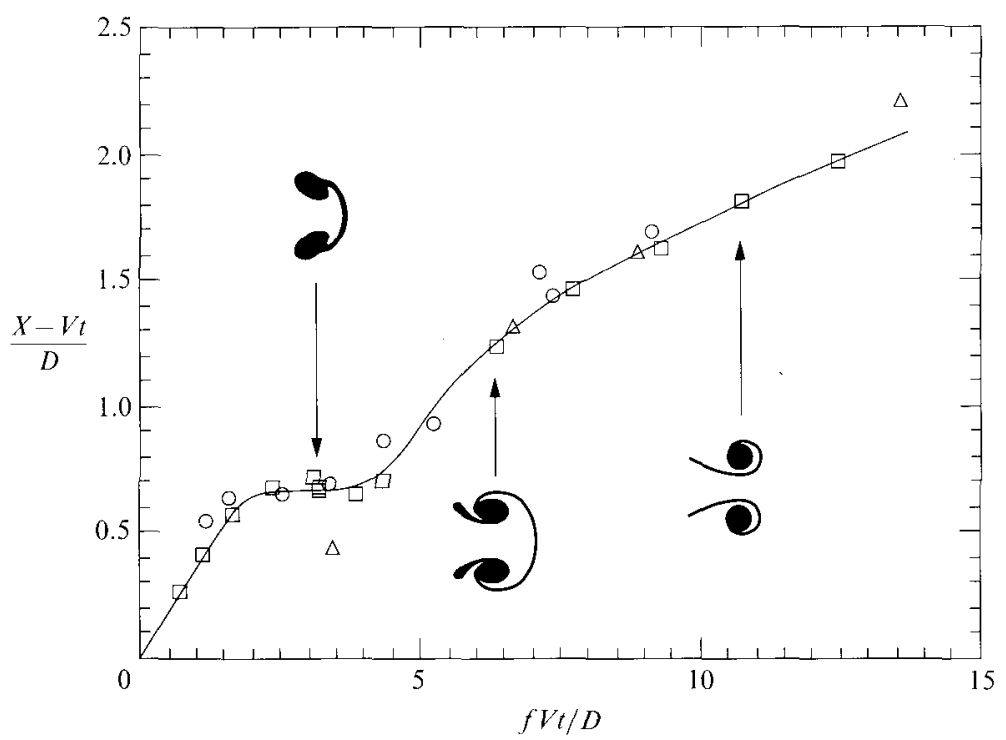

Figure 10. Relative displacement of the helium inhomogeneity for all three sets of runs, plotted against a timescale that collapses the data. $\bigcirc, M_{\mathrm{s}}=1.093, Z / D \mathrm{e}=3.2 ; \square, M_{\mathrm{s}}=1.093, Z / D_{\mathrm{c}}=4.8$; $\triangle, M_{\mathrm{s}}=1.15, Z / D_{\mathrm{e}}=4.8$.

The differences in the velocities measured at the two cross-sectional locations is a consequence of this tilting, and is, therefore, also caused by an axial density variation in the jet. Because helium is continuously diffusing out of the jet as it crosses the test section, the jet centreline density at the two cross-sectional locations may be significantly different. Note that the values of $U_{\mathrm{i}}$ and $U_{\mathrm{f}}$ given above were calculated assuming the jet core contained pure helium. The differences in the downstream displacements at the two spanwise locations strongly indicates that the jet centreline density is not uniform, and is, thus, greater than that of pure helium, at least at the location farthest from the jet exit $\left(Z / D_{\mathrm{e}}=4.8\right)$.

When the PLIF images taken at $Z / D_{\mathrm{e}}=3.2$ and 4.8 are further compared, one finds that not only do portions of the light-gas volume that lie closer to the jet exit travel downstream faster, but they deform at a greater rate as well. This is illustrated by figure 9 which compares images take at the two cross-sectional locations $Z / D_{\mathrm{e}}=$ 3.2 and 4.8. Although the two images appear to have been taken at precisely the same instant in time, figure $9(a)\left(Z / D_{\mathrm{e}}=3.2\right)$ was taken at $V t / D=3.62$, while figure $9(b)\left(z / D_{\mathrm{e}}=4.8\right)$ was taken at $V t / D=4.35$. A new timescale can be created (say $D / f V$ ) in which the two pictures in figure 9 can be made to occur at the same (new) dimensionless time. This is done by adjusting the parameter $f$ so that the new dimensionless time, $f V t / D$, is the same for both pictures. By choosing $f=1.20$ for the sequence with $M_{\mathrm{s}}=1.093$ and $Z / D_{\mathrm{e}}=3.2, f=1.00$ for $M_{\mathrm{s}}=1.093$ and $Z / D_{\mathrm{e}}=4.8$, and $f=1.03$ for $M_{\mathrm{s}}=1.15$ and $Z / D_{\mathrm{e}}=4.8$, the pictures from the three sets of runs can be made to match. Figure 10 is a plot of the relative displacement for all three sets of data, plotted against the new adjusted timescale. The data from all three sequences now fall on top of one another, forming a single trajectory, thus indicating that the deformation rate is directly related to the rate of translation.

Figure 10 shows that the terminal velocity of the vortex pair in the present experiments is proportional to $f V$. The circulation of each line vortex in a vortex pair is proportional to the product of the vortex spacing and its translational velocity, 


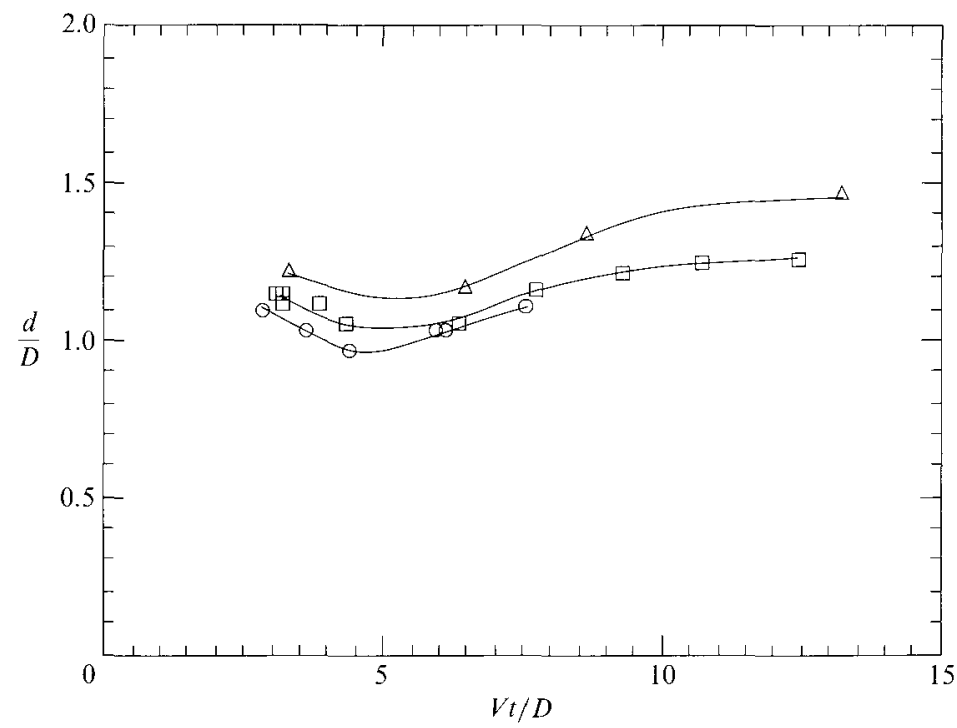

Figure 11. The time evolution of vortex spacing, for all three sets of runs. $O, M_{\mathrm{s}}=1.093$, $Z / D_{\mathrm{e}}=3.2 ; \square, M_{\mathrm{s}}=1.093, Z / D_{\mathrm{e}}=4.8 ; \triangle, M_{\mathrm{s}}=1.15, Z / D_{\mathrm{c}}=4.8$.

and is equal to the integral of vorticity over the half-plane containing the vortex. Therefore, the circulation of each vortex in the final stages of the experiments should be roughly proportional to $f V D$, and $f V D$ is, in some sense, a measure of the amount vorticity deposited by the shock wave on the cylinder boundary at a particular spanwise location. Hence, the factor $f$ may be viewed as an empirical parameter that adjusts for variations in the amount of vorticity produced by the shock interaction, that cannot be accounted for directly by changes in $V$ or $D$. The vortex circulation given by the model of Rudinger \& Somers is

$$
\Gamma=\frac{1-\sigma}{1+\sigma} V D
$$

Thus, according to this model, $f$ should be a function only of $\sigma$ and the variation of $f$ should be caused by differences in the jet centreline density at the two crosssectional locations. However, slightly different values of $f$ were found for runs taken at the same $Z$-location (thus having the same density), with different shock strengths $\left(M_{\mathrm{s}}=1.093\right.$ and 1.15), suggesting that $f$ is also a weak function of $M_{\mathrm{s}}$. Since neither compressibility nor viscous effects are accounted for in the model of Rudinger \& Somers, this dependence on shock strength may also be interpreted as a weak dependence on flow Mach or Reynolds numbers.

The PLIF images of figure 5 reveal a distortion process very similar to that displayed in the shadowgraph images of Haas \& Sturtevant (1987), and the computational results of Picone \& Boris (1988). However, when the past and present images are closely compared, the vortex spacing observed in figure 5 , appears to be significantly larger than that of the earlier experimental and computational results. Figure 11 shows the variation of vortex spacing with time for the present experiments. The vortex spacing $(d)$ was computed in the PLIF images where the portion of the image that is brighter than one half the brightest point in that image was clearly split into two pieces (i.e. figure $5 d$ and later). The distance (in the $y$ direction) between the centroids of each of these pieces was used as an indication of 


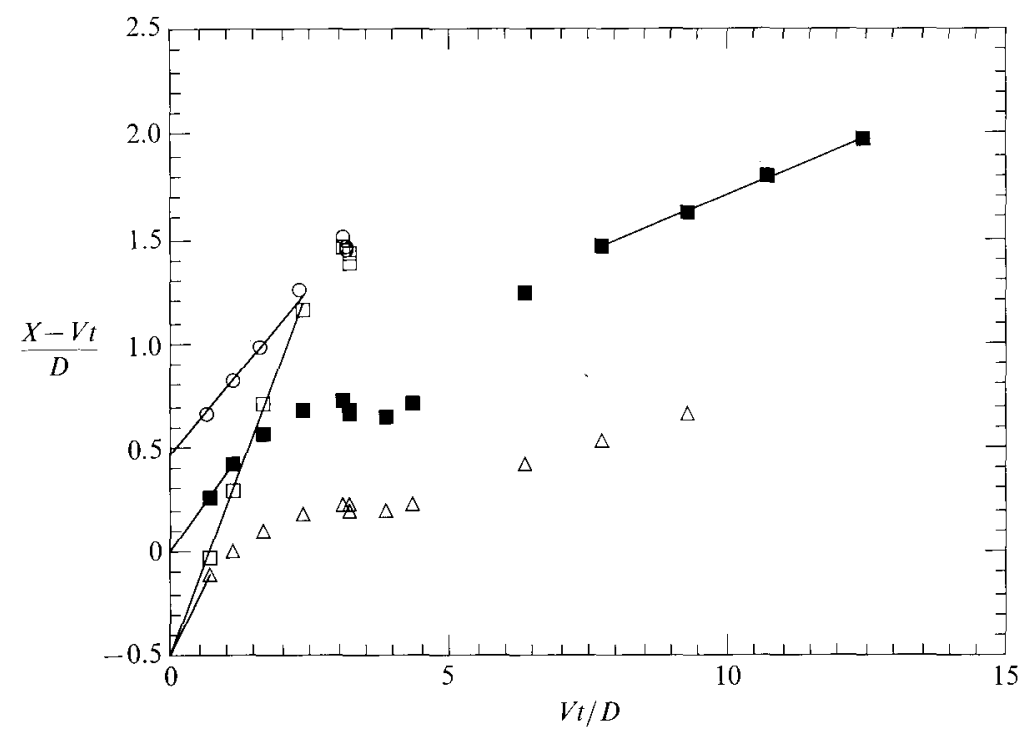

FigURe 12. Relative displacements of various points on the surface of the evolving helium inhomogeneity, for the set of runs with $M_{\mathrm{s}}=1.093$ and $Z / D_{\mathrm{e}}=4.8 . \triangle$, Cpstream edge; $\bigcirc$, downstream edge; $\square$, centre downstream edge; $\mathbf{Q}$, centroid.

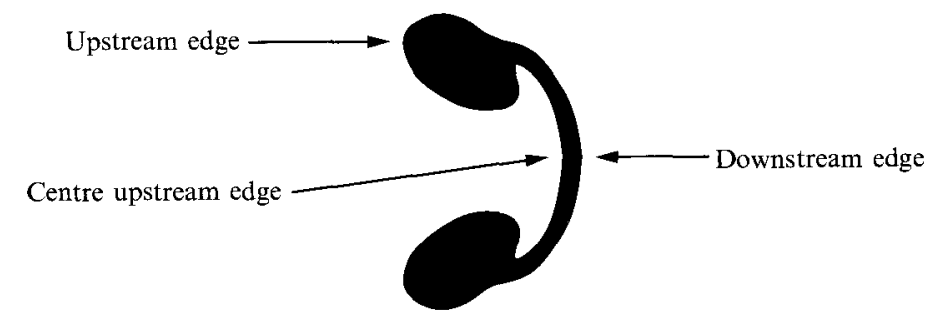

Figure 13. The locations of the characteristic points of figure 12 and table 1.

spacing, and is shown plotted in figure 11, scaled with jet diameter $D$. Note that for all three sets of runs, the spacing first decreases with time then increases until a nearly constant value is reached. Also note that the spacing is greater both for stronger shocks and for stations farther from the jet exit. The theory of Rudinger \& Somers gives a spacing ratio of $d / D=\frac{1}{4} \pi$, which is significantly less than the spacings given in figure 11. The vortex spacing in the present experiments is, therefore, larger than the previous experimental, computational and theoretical results. Because of the large difference in cylinder diameter between the present experiments $(0.66 \mathrm{~cm})$ and the experiments of Haas \& Sturtevant $(5 \mathrm{~cm})$, and the fact that the computational results of Picone \& Boris were inviscid, this disagreement may be attributed to Reynolds-number effects. However, the diffuse boundary in the present experiments could also have caused this difference.

The downstream displacement of several notable points on the helium/air interface, are shown plotted in figure 12. These points, which were first defined by Haas \& Sturtevant (1987) and are illustrated in figure 13, are the farthermost upstream and downstream edges of the deforming cylinder, and the centre upstream edge (what Haas \& Sturtevant call the 'air jet'). The downstream displacement of these points was obtained from the fluorescent images of the runs with $M_{\mathrm{s}}=1.093$ and $Z / D_{\mathrm{e}}=4.8$, and are shown plotted in figure 12 along with the displacement of 


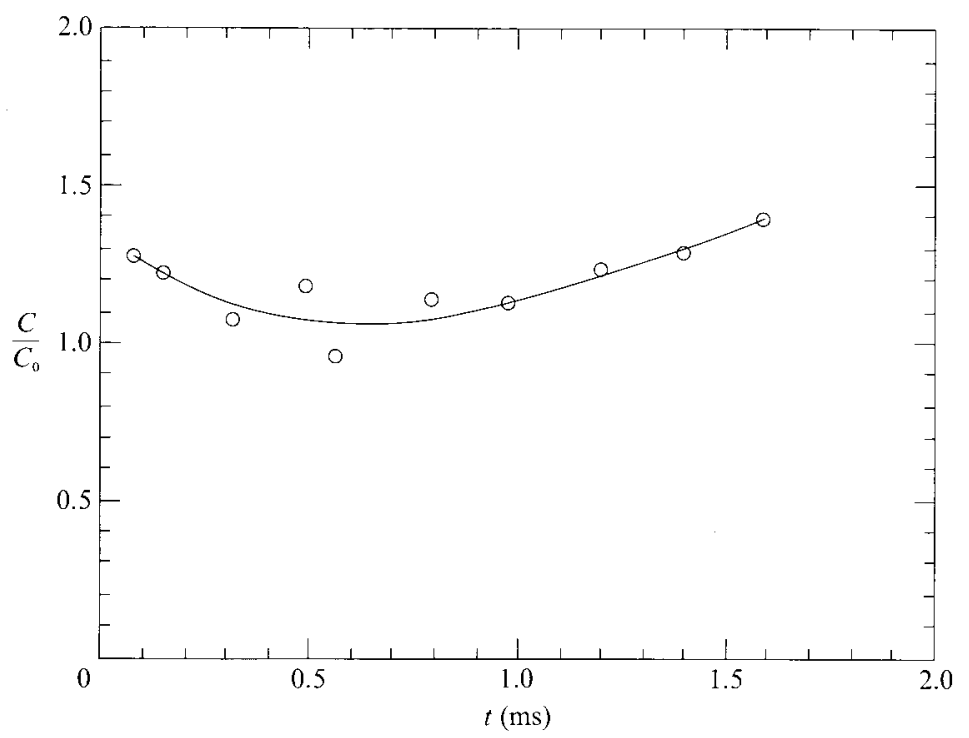

FIGURE 14. Maximum biacetyl concentration versus time, for the set of runs with $M_{\mathrm{s}}=1.093$ and $Z / D_{\mathrm{e}}=4.8$.

\begin{tabular}{|c|c|c|c|c|c|c|}
\hline & \multicolumn{2}{|c|}{ Present $\left(M_{\mathrm{s}}=1.093\right)$} & \multicolumn{2}{|c|}{ Haas \& Sturtevant } & \multirow{2}{*}{$\begin{array}{c}\text { Picone } \\
\& \text { Boris } \\
M_{\mathrm{s}}=1.22\end{array}$} & \multirow{2}{*}{$\begin{array}{l}\text { Rudinger \& } \\
\text { Somers }\end{array}$} \\
\hline & $Z / D_{\mathrm{e}}=\mathbf{4 . 8}$ & $Z / D_{\mathrm{e}}=3.2$ & $M_{\mathrm{s}}=1.085$ & $M_{\mathrm{s}}=1.22$ & & \\
\hline Upstream edge & 0.58 & - & 0.90 & 0.48 & 0.722 & 一 \\
\hline Downstream edge & 0.32 & - & 0.47 & 0.26 & 0.243 & - \\
\hline Centre upstream edge & 0.72 & - & 1.67 & 1.00 & 0.861 & - \\
\hline Centroid (initial) & 0.37 & 0.55 & - & - & $\ldots$ & 0.76 \\
\hline Vortex pair & 0.11 & 0.12 & 0.28 & 0.11 & 0.174 & 0.15 \\
\hline
\end{tabular}

TABLE 1. A comparison of some measured and computed dimensionless relative cylinder velocities, $(\mathrm{d} X / \mathrm{d} t-V) / V$

the centroid (i.e. the data of figure 7). Lines were fit to these trajectories using linear regression to obtain velocities. These curve fits are shown on figure 12 as solid lines, and the measured velocities are listed in table 1 along with values obtained by Haas \& Sturtevant (1987) and by Picone \& Boris (1988). Table 1 also contains initial and final velocities of the centroid, which may be compared with the theory of Rudinger \& Somers (1960), and the vortex-pair velocities measured by Haas \& Sturtevant and Picone $\&$ Boris. The present measurements are in reasonably good agreement with the findings of Haas \& Sturtevant and Picone \& Boris, falling for the most part within the range of the previously measured values.

\subsection{Mixing}

The fluorescence of biacetyl is linearly proportional to its concentration; therefore the PLIF images provide two-dimensional maps of the concentration of biacetyl contained within the accelerated inhomogeneity. Figure 14 is a plot showing how the maximum concentration of biacetyl within the inhomogeneity varies from time, for the set of runs with $M_{\mathrm{s}}=1.093$ and $Z / D_{\mathrm{e}}=4.8$. Each point in this plot represents the highest dye concentration in a fluorescent image, normalized by the highest 


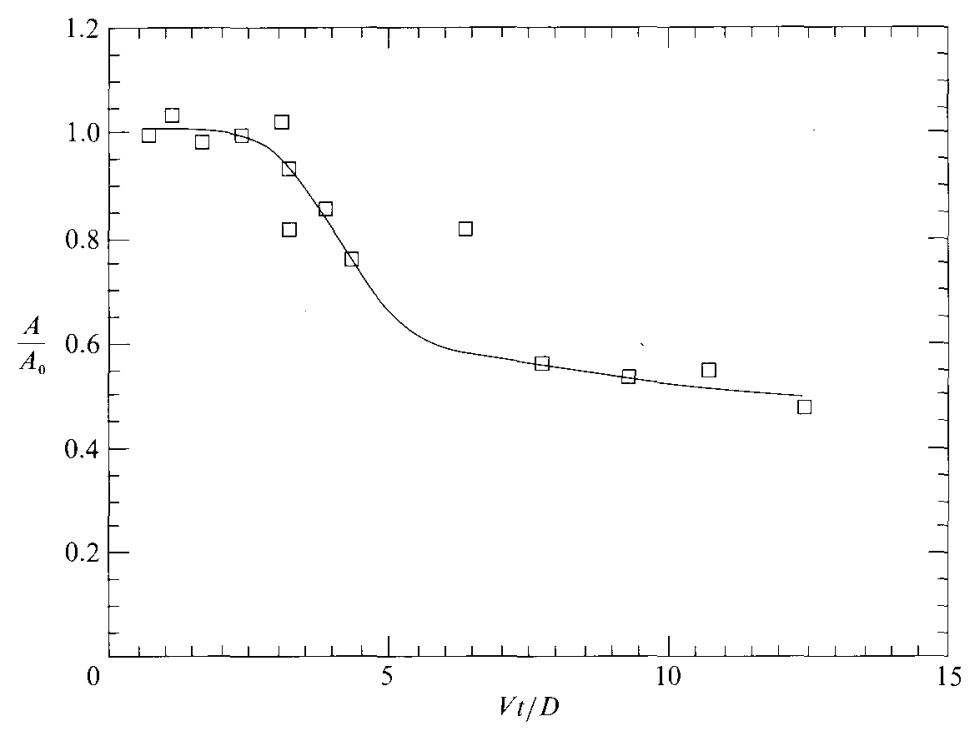

FIGURE 15. The time evolution of cross-sectional area of the helium inhomogeneity, for the set of runs with $M_{\mathrm{s}}=1.093$ and $Z / D_{\mathrm{e}}=4.8$.

concentration in the corresponding image of the initial jet. The scatter in the data shown in figure 14 is due to the inaccuracy of the PLIF system for making concentration measurements. It appears that the maximum image concentration initially decreases with time, then increases later on; nevertheless, it remains fairly constant throughout the evolution process.

When examining the images in figure 5, it is elear that the cross-sectional area of the inhomogeneity decreases markedly during this sequence. This decrease is a result of the mixing process causing the helium (and biacetyl) originally contained within the cylinder, to be dispersed into the surrounding air, thus decreasing the total volume of unmixed gas. This rapid decrease in volume is, therefore, an indication that mixing has occurred, and the rate at which the area enclosed by a contour (say at one half the original jet centreline concentration of biacetyl) decreases with time, provides a rough measure of the mixing rate. Since the highest concentration does not change significantly during this process, an equally good contour could be that at one half the maximum concentration in that particular fluorescent image. Because of the inaccuracy of the PLIF system when used for dye concentration measurements, this latter choice provides data with much less scatter. A plot of this area versus time is shown in figure 15 , for the sequence with $M_{\mathrm{s}}=1.093$ and $Z / D_{\mathrm{e}}=4.8$; and figure 16 is a plot containing data from all three sets of runs plotted using the timescale of figure 10. In these plots, area is scaled with that of the initial jet, adjusted to account for the compression of the shock wave. Note that the timescale that was used to collapse the displacement data of figure 10 does not collapse the mixing data of figure 16 .

Mixing takes place in these experiments in much the same way as it occurs in Marble's laminar flame model - by interfacial stretching, which steepens interfacial concentration gradients, and accelerates diffusion. The interaction of the shock wave with the helium cylinder propels the light gas through the surrounding air. The flow field resulting from this motion causes the interface at the front of the cylinder to be stretched, which steepens the concentration gradient at the front of the inhomo- 


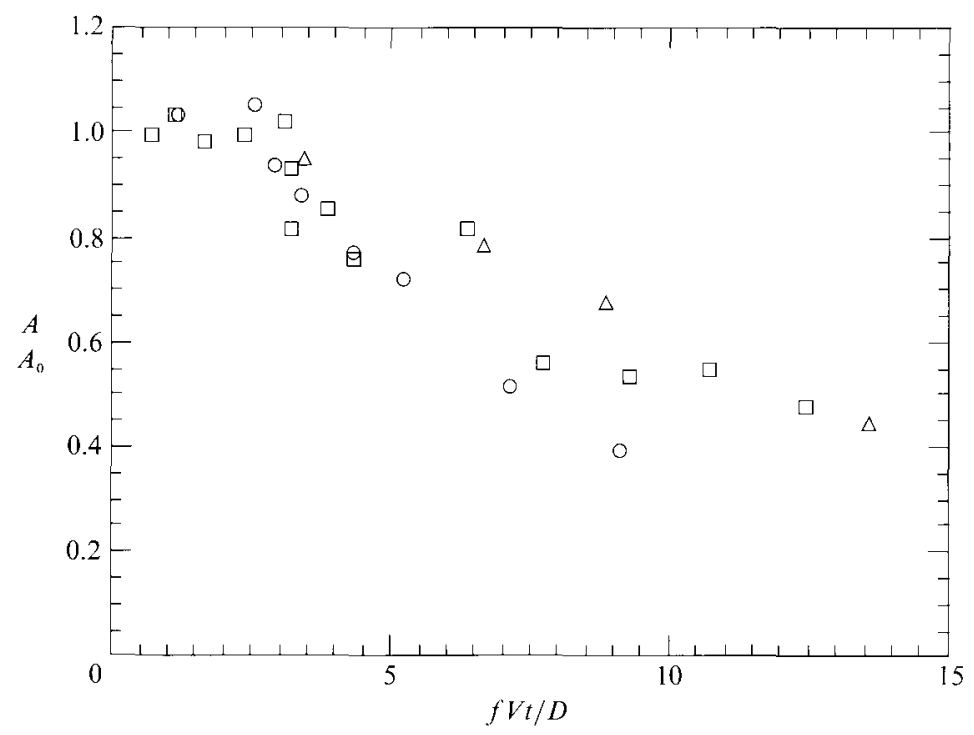

Figure 16. Cross-sectional areas for all three sets of runs, plotted against the timescale of figure 10. $\bigcirc, M_{\mathrm{s}}=1.093, Z / D_{\mathrm{e}}=3.2 ; \square, M_{\mathrm{s}}=1.093, Z / D_{\mathrm{e}}=4.8 ; \triangle, M_{\mathrm{s}}=1.15, Z / D_{\mathrm{e}}=4.8$.

geneity as observed in figure $5(b)$. Conversely, the interface at the rear of the cylinder is compressed, which broadens the gradient at the rear of the inhomogeneity. Eventually front and rear surfaces meet in front of the inhomogeneity and are both stretched by the motion induced by the developing vortex pair (figure $5 c-f$ ), causing the rapid disappearance of the strand of helium that connects the upper and lower halves of the inhomogeneity in figure $5(c)$. This process is further illustrated in figure $5(e-h)$, which shows the disappearance of the rearward portions of both the upper and lower halves of the inhomogeneity. In figure $5(e)$, each half of the inhomogeneity is further divided by the motion of a vortex sheet, to form a front and a back lobe. In the PLIF images that follow (figure $5 f-h$ ), the motion induced by the sheets stretches the back lobes, and as a result, the gas within the rearward regions dissipates until, in figure $\mathbf{5}(h)$, all that is left are thin strands of helium.

Marble (1985) obtained an expression for the consumption of fuel in the situation where a potential vortex lies on a boundary that separates fuel and oxidizer. He found the time $\left(t^{*}\right)$ required to consume all the fuel within a thin annulus, a radius $r$ from the core of a potential vortex, to be given by

$$
t^{*} \propto \frac{r^{2}}{\Gamma^{\frac{2}{3}} \mathscr{D}^{\frac{1}{3}}}
$$

where $\Gamma$ is the vortex circulation, and $\mathscr{D}$ is the molecular diffusivity. Marble's analysis addressed combustion; however, his model was based on a diffusion-limited reaction. Therefore, (5) applies equally well to the time it takes to mix the two fluids originally contained within the annulus. Since the present experiments involve mixing induced by the presence of vorticity, a scaling law similar to (5) should be applicable to the rate of helium dissipation observed in figures 15 and 16 . Defining a new mixing timescale $t_{\mathrm{m}}$ from $(5)$,

$$
t_{\mathrm{m}}=\frac{r^{2}}{\Gamma^{\frac{2}{3}} \mathscr{D}^{\frac{1}{3}}},
$$




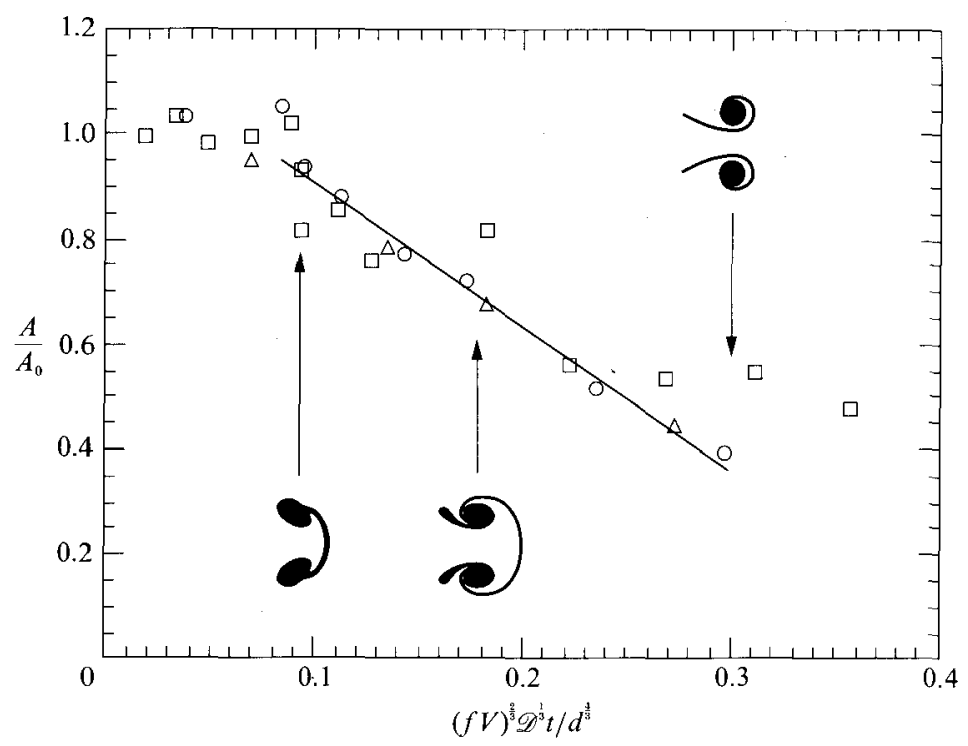

FIgURE 17. Cross-sectional areas for all three sets of runs, plotted against a timescale that collapses the data. The line represents the curve fit giving equation (8). $\bigcirc, M_{\mathrm{s}}=1.093, Z / D_{\mathrm{e}}=3.2 ; \square, M_{\mathrm{s}}$ $=1.093, Z / D_{\mathrm{e}}=4.8 ; \triangle, M_{\mathrm{s}}=1.15, Z / D_{\mathrm{e}}=4.8$.

and letting $\Gamma=f V d$ (a good approximation for the circulation of each vortex of the vortex pair) and $r=d$ (roughly the distance from the vortex cores where the mixing takes place), an appropriate timescaling for mixing in the present experiments might be

$$
\frac{t}{t_{\mathrm{m}}}=\frac{(f V)^{\frac{2}{3}} \mathscr{D}^{\frac{1}{3}} t}{d^{\frac{4}{3}}} .
$$

The data of figure 16 are replotted in figure 17 using the timescaling given in (7). This scaling greatly improves the collapse of the mixing data. Figure 17 shows that initially $\left(t / t_{\mathrm{m}}<0.1\right)$ mixing precedes slowly. This corresponds to the stage of development in which the originally circular cylinder is split into two lobes. Shortly thereafter, the mixing rate increases sharply. In this stage $\left(0.1<t / t_{\mathrm{m}}<0.25\right)$, the vortex sheets slice through the regions of helium, stretching the interface and accelerating the mixing process. The data in this region show that during this stage, the cross-sectional area decreases nearly linearly with time, indicating a constant mixing rate. At later times in figure $17\left(t / t_{\mathrm{m}}>0.25\right)$ the data with $M_{\mathrm{s}}=1.093$ and $Z / D_{\mathrm{e}}=4.8$ approach a constant value, indicating a decrease in mixing rate, while the areas of the other two cases continue to decrease. This stage corresponds to the development of the vortex pair. In this (terminal) stage of development the vortex sheets are almost completely wound upon themselves. After a period of time the diffusion of helium and vorticity acts to smooth out any large gradients in the vortex cores. In this situation very little mixing would occur, and one would expect the area to approach a constant value. The rate at which this final stage is reached is affected by the rate of diffusion and the relative thickness of the initial vortex sheets. Thus, one would expect the decrease in mixing to occur earlier at lower shock Mach number (giving more time for diffusion), and for cross-sections taken at a station farther from the jet exit (giving a thicker initial vortex sheet). This effect explains the differences in the mixing rates of the three cases shown in the later stages of figure 17. 
A linear fit to the data in the central part of figure 17 shows that the rate at which area decreases in this region is given by

$$
\frac{1}{A_{0}} \frac{\mathrm{d} A}{\mathrm{~d} t}=-2.76\left(\frac{(f V)^{\frac{2}{3}} \mathscr{D}^{\frac{1}{3}}}{d^{\frac{4}{3}}}\right) \approx-0.7 \times 10^{3} \mathrm{~s}^{-1},
$$

where $f$ varies from 1.0 to 1.2 as described above.

\section{Conclusions}

In these experiments, a cylindrical volume of a light gas was impulsively accelerated by a weak shock wave. A new technique was developed in which a laminar jet of helium was used to produce the light-gas cylinder, providing a flow that is unimpeded by the membranes used in previous work. In addition, the flow was visualized using planar laser-induced fluorescence instead of the shadowgraph technique used in earlier investigations. These improvements resulted not only in a higher quality of flow visualization, but also allowed for the measurement of species concentration, and hence the mixing that is produced by the shock/cylinder interaction.

The PLIF images obtained in the present experiments revealed a process very similar to that displayed in the shadowgraph images of Haas \& Sturtevant (1987) and by the computations of Picone \& Boris (1988). The displacement of points on the helium/air interface was measured, and agreed reasonably well with the findings of these earlier investigations. However, the vortex spacing in the present experiments was found to be significantly larger than that found in the previous experimental and computational results. The most likely cause for this difference is the effect of viscosity (or Reynolds number); however, other factors, such as the diffuse interface in the present experiments, may be responsible for the difference. It was found that portions of the inhomogeneity at the two cross-sectional locations, $Z / D_{\mathrm{e}}=3.2$ and 4.8 , travel downstream at different rates, and that the evolution process at these two locations proceeds at different rates as well. However, the displacement measurements for the runs taken at these two locations, as well as those taken with stronger shocks, could be collapsed onto a single trajectory by using a timescale that is linked to the rate of deformation, thus indicating that the rate at which the deformation proceeds is directly related to the rate of translation. This timescale is defined by an empirical parameter that was found by matching sequences of images at similar stages of evolution. The parameter accounts for differences in the vorticity deposited by the shock wave (at a particular spanwise location), and is for the most part a function of the difference in jet centreline density at the two cross-sectional locations; however, it may also be a weak function of the flow Mach or Reynolds numbers.

The primary objective of this research was to quantify the mixing induced by the interaction of a weak shock wave with a cylindrical volume of a light gas. It was found that rapid mixing takes place, in these experiments, as a result of motion induced by vorticity. This motion acts to stretch the boundaries that separate helium from air, which then steepens concentration gradients and accelerates mixing. This process is not all that different from what occurs in Marble's laminar flame model, in which a potential vortex induces the motion necessary for interfacial stretching and, thus, mixing. The area inside the contour at one half the highest concentration of biacetyl was found to decrease as the two gases are mixed; and the 
change in this area was used as a measure of mixing. It was found that, except for early and late stages in the mixing process, the time rate of change of this area is nearly constant, and given by equation (8). Note that this mixing rate is the result of a nominally two-dimensional laminar flow; and although mixing is produced, in these experiments, by a compressible object (a shock wave), the subsequent flow velocities were low enough to render the flow incompressible. If the shock strength were increased substantially one might expect a three-dimensional possibly turbulent flow, which would probably yield even higher mixing rates. However, increasing the shock strength could also result in a compressible flow, which would have an unknown effect on the mixing rate.

The author would like to acknowledge Professors F. E. Marble and E. E. Zukoski for initiating the study of shock-enhanced mixing, and the subsequent shock tube experiments to study the mixing of helium inhomogeneities, of which this research was a part. The author is indebted to Professors Marble and Zukoski as well as Professor B. Sturtevant for their help, guidance and support with this research. Credit is due to Dr. R. Miake-Lye for his help with the laser-induced fluorescence system, and to Dr L. Hill, and Professors A. Glezer and C. H. K. Williamson their helpful comments and suggestions during the preparation of this paper. This work was supported by the Air Force Office of Scientific Research contract F49620-C-0113, monitored by Dr J. Tishkoff. In addition, during 1987 and 1988 the author was supported by a Weizmann Fellowship.

\section{REFERENCES}

Bonazza, R., Broutllette, M., Goldstein, D., Haas, J.-F., Winckelmans, G. \& Stcrtevant, B. 1985 Rayleigh-Taylor instability of oblique interfaces. Bull. Am. Phys. Soc. 30, 1742.

Broadwfil, J. E. \& Breidentilat, R. E. 1982 A simple model of mixing and chemical reaction in a turbulent shear layer. J. Fluid Mech. 125, 397-410.

Broutilette, M. \& Sturtevant, B. 1988 Shock induced Rayleigh-Taylor instability at a continuous interface. Abstract submitted to the Intl Workshop on the Physics of Compressible Turbulent Mixing, 24-27 October 1988, Princeton, New Jersey.

Cowperthwarte, N. 1989 The interaction of a plane shock and a dense spherical inhomogeneity. Physica D 37, 264-269.

Epstein, A. H. 1974 Fluorescent gaseous tracers for three dimensional flow visualization. MIT Gas Turbine Lab Rep. 117.

EPSTEIN, A. H. 1977 Quantitative density visualization on a transonic compressor rotor. Trans. ASME A : J. Engng for Power 99, 460-475.

HaAs, J.-F. \& Sturtevant, B. 1987 Interaction of weak shock waves with cylindrical and spherical gas inhomogeneities. J. Fluid Mech. 181, 41-76.

Liepuann, H. W., Roshko, A., Coles, D. \& Sturtevant, B. 1962 A 17 -inch diameter shock tube for studies in rarefied gasdynamics. Rev. Sci. Instrum. 33, 625-631.

Marbie, F. E. 1985 Growth of a diffusion flame in the field of a vortex. In Recent Advances in the Aerospace Sciences (ed. Corrado Casci), pp. 395-413. Plenum.

Markstein, G. H. 1957 a Flow disturbances induced near a slightly wavy contact surface, or flame front, traversed by a shock wave. J. Aero. Sci. 24, 238.

Markstein, G. H. $1957 b$ A shock tube study of flame front-pressure wave interactions. 6th Intl Symp. Combust., pp. 387-398. Reinhold.

Meshkov, E. E. 1969 Instability of the interface of two gases accelerated by a shock wave. Izv. Akad. Nauk. SSSR Mekh. Zhidk. Gaza 4, 151-157 [Russian: Izv. Acad. Sci. USSR Fluid Dyn. 4, 101-104].

PJCONE, J. M. \& BoRIS, J. P. 1988 Vorticity generation by shock propagation through bubbles in a gas. J. Fluid Mech. 189, 23-51. 
Ptcone. J. M., Oran, E. S., Boris, J. P. \& Young, T. R. 1985 Theory of vorticity generation by shock wave and flame interactions. In Dynamics of Shock Waves, Explosions, and Detonations, pp. 429-448. AIAA.

Rich'myer, R. D. 1960 Taylor instability in shock acceleration of compressible fluids. Commun. Pure Appl. Maths 23, 297-319.

RCDinger, G. 1958 Shock wave and flame interactions. Combustion and Propulsion, Third $A G A R D$ Colloq. London. Pergamon.

R.DINGER, G. \& Somprs, L. I. 1960 Behaviour of small regions of different gases carried in accelerated gas flows. J. Fluid Mech. 7, 161-176.

SaffMan, P.G. \& VeIRon, D. I. 1989 Kinetic energy generated by incompressible RichtmyerMesbkov instability in a continuous stratified fluid. Phys. Fluids A 1, 1767-1771.

IANG, X., ZABCsky, N. J. \& ChEN, I. L. 1990 'Breakthrough' via dipolar-vortex/jet formation in shock-accelerated density-stratified layers. Phys. Fluids A 2, 892-895.

Zaitsev. S. G., Lazareva, E. V., Chernlkha, V. V. \& Belyaev, V. M. 1985 Experimental investigation of the hydrodynamic instability of the interface between media of different density in an acceleration field. Translated from Teplofizika Vysokikh Temperatur 23, 535-541. 